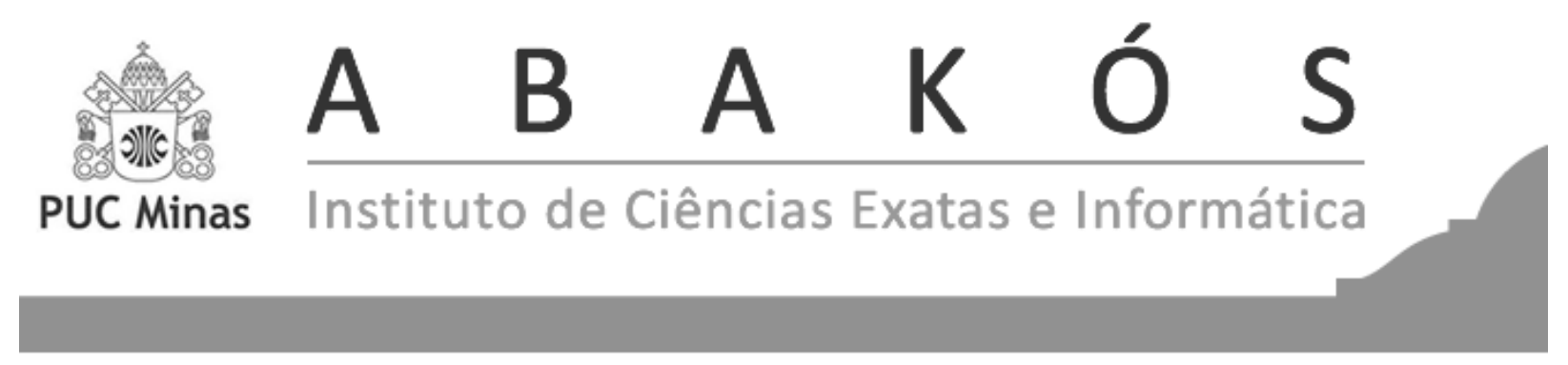

\title{
Discalculia: Um Mapeamento de Artigos Brasileiros*
}

Dyscalculia: A Brazilian Articles Mapping

\author{
Lanúzia Almeida Brum Avila ${ }^{1}$ \\ Isabel Cristina Machado de Lara ${ }^{2}$
}

\begin{abstract}
Resumo
Este artigo apresenta o mapeamento teórico de artigos que abordam a temática Discalculia, transtornos de aprendizagem em Matemática (TAM) e dificuldades de aprendizagem em Matemática (DAM) publicados em periódicos acadêmicos brasileiros. Tem como objetivo verificar o estado da arte dessas produções, buscando por suas convergências e divergências em relação a alguns aspectos, entre eles o modo como são conceituados os termos Discalculia, TAM e DAM e as contribuições aos profissionais da área da Educação. Para selecionar as produções brasileiras dos últimos anos, utilizou-se o banco de periódicos disponibilizados pela Coordenação de Aperfeiçoamento de Pessoal de Nível Superior (CAPES) e a Scientific Eletronic Library Online (SCIELO). A análise evidencia que os artigos divergem quanto aos termos adotados na literatura para referir-se às dificuldades de aprendizagem (DA) e aos transtornos de aprendizagem (TA), fazendo uso não somente do termo Discalculia, como também DAM, TAM e distúrbios de aprendizagem, o que acaba por dificultar a comparação entre os dados pesquisados, pela falta de padronização quanto às denominações utilizadas.
\end{abstract}

Palavras-chave: Discalculia. Transtornos de Aprendizagem em Matemática. Dificuldades de Aprendizagem em Matemática.

\footnotetext{
* Submetido em 05/05/2016 - Aceito em 04/09/2017

${ }^{1}$ Mestre em Educação em Ciências e Matemática pela PUCRS. Licenciada em Pedagogia Orientação Educacional pela FAPA Psicopedagoga Institucional e Clínica pela FAPA- lanuzia.avila@acad.pucrs.br

${ }^{2}$ Doutora e Mestre em Educação pela UFRGS. Licenciada em Matemática pela UFRGS- isabel.lara@ pucrs.br
} 


\begin{abstract}
This article presents the theoretical mapping of articles from Brazilian academic journals, which deal with the issue of Discalculia, learning disorders in Mathematics (TAM) and learning difficulties in Mathematics (DAM). The objective is to verify the state of the art of these productions, seeking for their convergences and divergences in relation to some aspects such as the way how the terms Discalculia, TAM and DAM and contributions to the professionals in the field of Education are conceptualized. In order to select the Brazilian productions in the last years, it was used the database of periodicals journals provided by the Coordination of Improvement of Personnel of Superior Level (CAPES) and Scientific Eletronic Library Online (SCIELO). Based on the analysis, it shows that the articles diverge in terms adopted in the literature referring to learning difficulties (DA) and learning disorders (TA), not only using the term Discalculia, but also DAM and TAM and Learning disturbances, which makes it difficult to compare the data surveyed, due to the lack of standardization of the denominations used.
\end{abstract}

Keywords: Dyscalculia. Learning Disorders in Mathematics. Learning difficulties in Mathematics. 


\section{INTRODUÇÃO}

Em 2013, iniciou-se o Projeto Avaliação de Crianças em Risco de Transtornos de Aprendizagem (ACERTA), apoiado pela Coordenação de Aperfeiçoamento de Pessoal de Nível Superior (CAPES), no Instituto do Cérebro do Rio Grande do Sul da Pontifícia Universidade Católica do Rio Grande do Sul (INSCER/PUCRS).

O projeto ACERTA surgiu com o propósito de melhor entender as mudanças que ocorrem no cérebro das crianças em fase de alfabetização. O principal objetivo é de compreender por que algumas crianças desenvolvem transtornos de aprendizagem. Em conjunto com outros dois centros de pesquisa, em Florianópolis e Natal, estão sendo identificados biomarcadores precoces desses transtornos, através do uso da neuroimagem funcional e estrutural (BUCHWEITZ, 2015).

Esse projeto é formado por pesquisadores de diferentes áreas: Psicopedagogia, Fonoaudiologia, Neurologia, Neuropsicologia, Matemática e Letras. Ao ingressar nessa equipe, a Dra. Isabel Cristina Machado de Lara e a mestranda Letícia da Silva Pimentel iniciaram o desenvolvimento de uma pesquisa sobre o transtorno da aprendizagem em Matemática (TAM), mais conhecido como Discalculia, concluindo, em março de 2015 a pesquisa intitulada "Discalculia e Provinha Brasil: uma análise do desempenho de estudantes do primeiro ano"; que apresenta resultados importantes para a continuação da dissertação "Avaliação e intervenções psicopedagógicas em crianças com indícios de Discalculia”.

Para reafirmar os estudos acerca da Discalculia, em 2013, constituiu-se o Grupo de Pesquisas e Estudos sobre Discalculia (GEPED/PUCRS), coordenado pela Dra. Isabel Cristina Machado de Lara. Assim, iniciou-se uma nova etapa, com diferentes pesquisas desenvolvidas pelos integrantes do grupo ${ }^{3}$.

Nos últimos anos, com base na experiência vivenciada ${ }^{4}$ em atendimentos psicopedagógicos, as dificuldades de aprendizagem (DA), apresentadas por alguns estudantes, são perceptíveis desde a Educação Infantil até o Ensino Médio. Muitas das dificuldades apresentadas iniciam-se na construção do número, adição, subtração, multiplicação, divisão, resolução de problemas, cálculos mentais, frações, raciocínio lógico, dentre outros.

Nem sempre é possível o professor detectar se a dificuldade do estudante é específica de um conteúdo ou de uma disciplina, ou se o mesmo apresenta dificuldades em outras disciplinas. Em alguns casos, se faz necessário o auxílio do setor pedagógico da escola, almejando a busca de informações sobre a história de vida desse estudante, por meio de uma conversa com os pais. Nesses casos, é necessário identificar se essa dificuldade é específica ou se está relacionada a fatores secundários, de modo que o professor possa intervir adequadamente e a aprendizagem venha a acontecer com êxito.

De acordo com Butterworth (2005), as DAM são menos estudadas do que as dificuldades no aprendizado da leitura e da escrita, em particular a Dislexia, havendo poucas pesquisas no

\footnotetext{
${ }_{3}^{3}$ Ambas autoras integram o GEPED/PUCRS.

${ }^{4}$ Uma das autoras é psicopedagoga tendo diferentes experiências acerca do atendimento de crianças com DA e TA.
} 
meio médico e educacional acerca do assunto, dificultando a avaliação psicopedagógica pela falta de instrumentos capazes de proporcionar com precisão se a criança é portadora ou não de Discalculia.

Severe difficulties in learning about numbers and arithmetic are probably as widespread as disorders of literacy development (dyslexia). The best prevalence estimates for each lie between $3.6 \%$ and $6.5 \%$ [...]. Studies in the U.K. have revealed that poor mathematical skills are more of a handicap in the workplace than poor literacy skills (Bynner \& Parsons, 1997). However, there has been much less research on dyscalculia than on dyslexia, and it is a much less widely recognized type of learning disability. In the U.K., its existence was first recognized by the Department of Education in 2001 (DfES, 2001). ${ }^{5}$ (BUTTERWORTH, 2005, p. 455).

Confirmando essa ideia, no caso do Brasil, Pimentel e Lara (2013) realizaram um mapeamento de dissertações e teses brasileiras, evidenciando que:

Em uma análise parcial, evidencia-se que embora muitos estudos sejam feitos relacionados a distúrbios de aprendizagem, apenas 4,4\% desses trabalhos mencionam distúrbios de aprendizagem na Matemática. Da mesma forma, quando trata-se de transtornos de aprendizagem, apenas, aproximadamente, 7,9\% referem-se à Matemática. Esses dados podem ser um indicativo de que pouco se discute sobre esse tema, principalmente no âmbito da Educação Matemática. (PIMENTEL; LARA, 2013, p. 10).

Adicionado a isso, percebemos em nossas pesquisas, que os testes existentes ainda não conseguem por si só contemplar a avaliação das habilidades em defasagem em cada uma das categorias de Discalculia definidas por Kosc (1974), sejam elas: discalculia verbal; discalculia practognóstica; discalculia léxica; discalculia gráfica; discalculia ideognóstica; discalculia operacional.

Pensando nessa problemática, elaborou-se um mapeamento teórico de artigos de periódicos acadêmicos brasileiros que abordam a temática Discalculia, TAM ou dificuldades de aprendizagem em Matemática (DAM).

O objetivo desse mapeamento é verificar o estado da arte dessas produções, buscando por suas convergências e divergências em relação a alguns aspectos como o modo como são conceituados os termos Discalculia, TAM e DAM e as contribuições dessas produções aos profissionais da área da Educação e da Saúde.

Para tanto, realizou-se uma busca ao banco de periódicos disponibilizados pela CAPES e pela Scientific Eletronic Library Online (SCIELO). Os critérios de seleção usados foram as palavras chaves: Discalculia, TAM e DAM. Em um primeiro momento, foi realizada a leitura

\footnotetext{
${ }^{5}$ Dificuldades graves na aprendizagem sobre números e aritmética são tão prováveis como os distúrbios do desenvolvimento da alfabetização (dislexia). As melhores estimativas de prevalência para cada uma delas situam-se entre 3,6\% e 6,5\% [...]. Estudos na U.K. revelaram que habilidades matemáticas fracas são mais uma desvantagem no local de trabalho do que uma fraca capacidade de alfabetização (Bynner \& Parsons, 1997). No entanto, tem havido muito menos pesquisas sobre a discalculia do que sobre a dislexia, e esse tipo de deficiência de aprendizagem é menos amplamente reconhecida. Na U.K., sua existência foi reconhecida pela primeira vez pelo Departamento de Educação em 2001 (DfES, 2001). (tradução nossa, 2017).
} 
dos resumos e, posteriormente, foram selecionados 10 artigos de periódicos para análise integral, com o objetivo de analisar convergências e divergências entre os periódicos, classificando e agrupando as produções, por meio de síntese das ideias fundamentais de cada uma delas. Para realizar a análise, elencaram-se os seguintes aspectos: objetivos; referências teóricas utilizadas; procedimentos metodológicos adotados; resultados encontrados; principais considerações; contribuições educacionais.

\section{CONCEITOS E DEFINIÇÕES}

De acordo com Biembengut (2008), é relevante entender os conceitos e definições acerca dos termos envolvidos na pesquisa. A compreensão dos termos utilizados auxiliará na identificação dos que foram utilizados e a elaboração de outros conceitos e definições ou na adoção de algum deles, com o qual tem-se a intenção de conduzir a pesquisa.

Em particular, no caso deste artigo, isso é necessário, pois existe uma diversidade de termos relativos à Discalculia. Sendo assim, torna-se relevante antes de iniciar o mapeamento dessas produções, apresentar diferentes terminologias, definições e concepções utilizadas pelos teóricos e pesquisadores que tratam do assunto. Os principais termos referidos ao longo do mapeamento são: Discalculia, TAM e DAM.

Tem-se como propósito apresentar subsídios teóricos que contribuam para a compreensão acerca do TAM, conhecido como Discalculia. Para isso, a presente seção abordará os seguintes aspectos: Dificuldades e transtornos no processo de ensino e aprendizagem; Discalculia do Desenvolvimento; Procedimentos de avaliação.

\subsection{Dificuldades e transtornos no processo de ensino e aprendizagem}

Os termos utilizados para denominar as DA vêm se modificando ao longo dos anos. Contudo, de acordo com a Isley (2015) Classificação Estatística Internacional de Doenças e Problemas Relacionados à Saúde - CID-10 (1993) e o DSM-5 (2014) Manual Diagnóstico e Estatístico de Transtornos Mentais - DSM-V (2014), são empregados diversos termos para descrever DA, às vezes definida como desordem de aprendizagem ou TA, em que o indivíduo apresenta dificuldades em aprender adequadamente. Em consequência disso, é relevante apresentar diferentes terminologias, definições e concepções acerca dos termos DA e TA.

De acordo com a CID-10, os transtornos de aprendizagem:

[...] são transtornos nos quais os padrões normais de aquisição de habilidades são perturbados desde os estágios iniciais do desenvolvimento. Eles não são simplesmente uma consequência de uma falta de oportunidade de aprender nem são decorrentes de qualquer forma de traumatismo ou de doença cerebral adquirida. Ao contrário, pensa-se que os transtornos originam-se de anormalidades no processo cognitivo, que derivam em grande parte de algum tipo de 
disfunção biológica. (1993, p. 236).

E de acordo com o DSM-5:

Um Transtorno Mental é uma síndrome caracterizada por perturbação clinicamente significativa na cognição, na regulação emocional ou no comportamento de um indivíduo que reflete uma disfunção nos processos psicológicos, biológicos ou de desenvolvimento subjacentes ao funcionamento mental (2014, p. 20).

Ohlweiler (2006) menciona que: "Os termos utilizados, tais como "distúrbios", "dificuldades", "problemas", "discapacidades", "transtornos", são encontrados na literatura e, muitas vezes são empregados de forma inadequada.” A autora (2006) salienta, ainda, que se faz necessário estabelecer diferenças entre dificuldades e transtornos da aprendizagem.

Na perspectiva de Rotta (2006), as DA podem ser definidas como alterações heterogêneas de problemas, os quais alteram as possibilidades da criança aprender, independentemente de suas questões neurológicas. Em se tratando do TA, refere-se como sendo dificuldades primárias ou específicas, decorrentes de alterações no Sistema Nervoso Central (SNC).

No mesmo sentido, conforme Relvas (2011), as DA são definidas como “[...] resultado de algumas falhas intrínsecas ou extrínsecas desse processo aprendizagem [...]" (2011, p. 58). Além disso, abrangem "[...] um grupo heterogêneo de problemas capazes de alterar as possibilidades de a criança aprender, independentemente de suas condições neurológicas para fazê-lo." (RELVAS, 2011, p. 58).

Na mesma perspectiva, Fonseca (2000) refere-se às DA como sendo desordens decorrentes das dificuldades significativas referentes à audição, leitura, escrita, fala e raciocínio matemático. O problema de aprendizagem é intrínseco ao indivíduo, que apresenta potencial intelectual normal e mesmo, diante de sua inteligência, seu rendimento escolar está abaixo do esperado. Conforme o autor (2000, p. 85): “Em geral, a criança ou jovem com DA apresenta QI dentro ou acima da média, todavia revela um aproveitamento escolar abaixo dela em algumas áreas, mas não em outras.".

Complementando essa ideia, utiliza Ohlweiler (2006), que ressalta:

As dificuldades de aprendizagem podem ser [...] de percurso, causadas por problemas da escola e/ou da família, que nem sempre oferecem condições adequadas para o sucesso da criança [...] dificuldades que a criança pode apresentar em alguma matéria ou em algum momento da vida, além de problemas psicológicos, como falta de motivação e baixa auto-estima (OHLWEILER, 2006, p. 127).

Fernández (2001) fortalece essa definição, evidenciando que muitas crianças no período escolar tendem a apresentar dificuldades na realização das tarefas escolares, podendo, em alguns casos, serem advindas da proposta pedagógica do professor, problemas relativos a questões familiares ou déficits cognitivos. A autora (2001) denomina esses fatores como sendo dificuldades de percurso, decorrentes da falta de condições apropriadas para que a aprendizagem ocorra com êxito. 
Referente à aprendizagem, Fernández (2001) sobreleva o papel que exerce a escola no processo de aprendizagem, pois o modo que o docente reconhece o estudante como sujeito da aprendizagem, o espaço que é proporcionado ao perguntar, a estimulação por meio do lúdico, a socialização com os colegas da mesma idade e a maneira como o estudante autoriza-se como autor de seu processo de autoria de pensamento, são fundamentais para o aprendizado.

Em específico a respeito da Matemática, Machado (1997) refere-se como uma disciplina básica no currículo das escolas e presente desde os anos iniciais da escolarização, em todo o mundo, pelo consenso de sua necessidade no ensino desde o processo da alfabetização. Todavia, muitos docentes desconhecem a utilidade da Matemática e a necessidade de contextualizá-la, sem vinculá-la, assim, à realidade dos estudantes.

Conforme Lara (2004), a Matemática é vista por alguns estudantes como uma das disciplinas mais difíceis do currículo escolar. Para alguns professores, é historicamente natural que existam dificuldades nessa disciplina, o que acaba levando a constituição de um discurso tido como verdadeiro em muitas escolas, implicando em um ensino inapropriado da Matemática.

Desse modo, Lara (2004) relata que é preciso colocar esse discurso, dito como verdade absoluta, sob suspeita, pois assim como existem dificuldades para o estudante aprender, também existe a causa para que isso aconteça. Em muitos casos, segundo a autora (2004), essas dificuldades estão relacionadas ao ensino ultrapassado e descontextualizado da realidade dos estudantes.

Corroborando essa ideia, Vieira (2004) especifica que:

Muitas vezes, o aluno enfrenta [...] DAM, em razão de lhe "ensinarem" através da memória mecânica, sem nunca estimularem a aprendizagem a partir da assimilação dos conceitos básicos, tais como classificação, ordenação, sequência e ordenação, correspondência, conservação (VIEIRA, 2004, p. 110).

Lara (2004) afirma que a maneira como o docente se expressa ao ministrar suas aulas, pode influenciar negativamente e tornar-se um dos indícios das DAM para muitos estudantes. Para a autora (2004), é importante o docente estar atento ao comunicar-se com seus alunos, propiciando a compreensão do que está sendo dito, para que possam abstrair determinado assunto e conseguir relacioná-lo aos conhecimentos já existentes.

García (1998) evidencia que, nas DAM, se faz necessário compreender como ocorrem os processos e o desenvolvimento das habilidades de aprendizagem da Matemática nas crianças. Em particular, faz-se necessário compreender quais são as habilidades matemáticas envolvidas nos dois transtornos relacionados à aprendizagem em Matemática mais conhecidos, a Acalculia e a Discalculia.

\subsection{Discalculia do Desenvolvimento}

Conforme Myklebust e Jhonson (1962), a Discalculia do Desenvolvimento foi definida por Cohn (1961 apud MYKLEBUST; JHONSON, 1962) e Gerstmann (1940 apud MYKLE- 
BUST; JHONSON, 1962), como uma deficiência na aprendizagem da Matemática relacionada ao reconhecimento e manipulação dos numerais, provocada por uma disfunção no cérebro. Bakwin (1960 apud KOSC, 1974) corrobora, definindo Discalculia como uma dificuldade em contar.

Myklebust e Johnson (1962), em uma tentativa de sistematizar a definição de Discalculia, ressaltam brevemente as dificuldades na Matemática ocasionadas pelo respectivo transtorno, evidenciando que:

[...] In school children this condition often is manifested by an obvious disability in arithmetic although intelligence and other aspects of behavior are well within the normal range. Some are deficient in arithmetical concepts, some cannot read numbers and hence, cannot write them. Others cannot associate meaning with the symbols which indicate the arithmetical process to be employed, such as $=, X$ and $+.1^{6}$ (MYKLEBUST; JHONSON, 1962, p. 14).

De acordo com Kosc (1974), Gerstmann (1957 apud KOSC, 1974) estabelece como definição para a Discalculia, a inabilidade em relação às operações simples e/ou complexas, dificuldade em compreender a orientação da sequência dos numerais e também quanto às frações. Com base nesses estudos, Kosc (1970 apud KOSC, 1974) apresenta sua definição de Discalculia do Desenvolvimento:

Developmental dyscalculia is a structural disorder of mathematical abilities which has its origin in a genetic or congenital disorder of those parts of the brain that are the direct anatomico-physiological substrate of the maturation of the mathematical abilities adequate to age, without a simultaneous disorder of general mental functions.1 (KOSC, 1970, p. 192 apud KOSC, 1974, p. $165)^{7}$

Na mesma perspectiva, Haase, Costa, Michelli, Oliveira e Wood (2011) afirmam que a Discalculia é uma entidade nosológica, a qual compromete as habilidades relacionadas ao processamento numérico e cálculos envolvendo operações simples. Para os autores (2011), trata-se de um transtorno de aprendizagem da aritmética, uma dificuldade crônica em aprender Matemática afetando entre 3\% e 6\% dos estudantes no período escolar. Os autores (2011) enfatizam que estudantes com esse transtorno tendem a apresentar problemas quanto ao conceito dos numerais, habilidades de contagem em defasagens, dificuldades relacionadas à transcodificação numérica e problemas na resolução de cálculos envolvendo as quatro operações.

Muitas vezes, já na Educação Infantil, é possível perceber os primeiros sinais dos sintomas, tais como: dificuldade em contar; usar adequadamente conceitos matemáticos (mais e

\footnotetext{
${ }^{6}$ Em crianças em idade escolar esta condição muitas vezes se manifesta por uma incapacidade em aritmética, embora a inteligência e outros aspectos do comportamento estão dentro de um intervalo normal. Alguns conceitos em aritmética são deficientes, alguns não podem ler numerais e, portanto, não podem escrevê-los. Outros não podem associar o significado com os símbolos que indicam o processo aritmético a ser empregado, como =, $\mathrm{x}$ e $+($ tradução nossa, 2017).

${ }^{7}$ Discalculia do Desenvolvimento é uma disfunção estrutural de habilidades matemáticas, que tem sua origem numa deficiência genética ou congênita dessas partes do cérebro, que são os substratos anatômico-fisiológicos diretos da maturação das habilidades matemáticas de acordo com a idade, sem uma disfunção simultânea de funções mentais gerais (tradução nossa, 2017).
} 
menos, pouco ou muito, antes e depois); as noções de tempo e de espaço tendem a ser problemáticas para os estudantes discalcúlicos (HAASE et al., 2011).

Além disso, Bastos (2006) complementa, afirmando que a Discalculia do Desenvolvimento, pode ser vista como "[...] uma dificuldade em aprender matemática, com falhas para adquirir proficiência adequada neste domínio cognitivo, a despeito de inteligência normal, oportunidade escolar, estabilidade emocional e motivação necessária [...].”(BASTOS, 2006, p. 202). Kocs (1974, p. 167-168) propôs um sistema uniforme para denominar e classificar as disfunções quanto às habilidades matemáticas em defasagem na Discalculia, classificando-as em seis categorias.

- Discalculia verbal: dificuldade quanto às habilidades verbais, quanto aos termos e símbolos matemáticos, bem como: nomear quantidades e numerais; reconhecer os símbolos matemáticos.

- Discalculia practognóstica: dificuldade na manipulação de objetos ou figuras, quanto a enumerar e comparar quantidades.

- Discalculia léxica: dificuldade em relação à leitura dos símbolos matemáticos, bem como as operações matemáticas e seus respectivos sinais, dígitos e numerais.

- Discalculia gráfica: dificuldade em escrever os símbolos matemáticos.

- Discalculia ideognóstica: dificuldade em relação à compreensão de conceitos matemáticos e quanto à execução das operações mentais.

- Discalculia operacional: dificuldades na resolução de operações.

Vale ressaltar que Haase et al. (2011, p. 273) enfatizam a necessidade da "[...] caracterização de diferentes subtipos ainda merece muita investigação. Há muito a fazer pela caracterização da DC com vistas a diminuir o impacto negativo grave da discalculia sobre o desenvolvimento infantil.".

Uma definição mais atual de Discalculia encontra-se no DSM-V (2014, p. 67): “[...] um termo alternativo usado em referência a um padrão de dificuldades caracterizado por problemas no processamento de informações numéricas, aprendizagem de fatos aritméticos e realização de cálculos precisos ou fluentes.".

Os sintomas evidenciados na Discalculia, de acordo com Bastos (2006), estariam relacionados a alguns fatores: erro na formação dos numerais, os quais, na maioria das vezes, são representados invertidos; comorbidade com Dislexia; falta de habilidade para calcular somas simples; inabilidade quanto ao reconhecimento de sinais das operações; dificuldade em ler valores dos numerais; problemas quanto à memorização de fatos numéricos básicos; dificuldades no transporte dos numerais em cálculos; problemas quanto à ordenação e espaçamento entre os numerais em cálculos envolvendo multiplicação e divisão.

Na perspectiva de Vieira (2004), alunos com Discalculia tendem a apresentar dificuldades em relação à leitura e à escrita dos numerais, ao reconhecimento dos símbolos numéricos, 
aos sinais das operações, à resolução de cálculos simples envolvendo adição e subtração e à localização em operações envolvendo multiplicação e divisão. Alunos discalcúlicos tendem a apresentar problemas em relação a memória. As dificuldades são acentuadas, estando demasiadamente abaixo do esperado e dificultando, assim, a execução dos cálculos mentais.

Vieira (2004) destaca a importância do professor estar preparado e saber diferenciar os sintomas relacionados às DAM e à Discalculia, referindo-se às seguintes manifestações da Discalculia:

\footnotetext{
"Dificuldade na identificação de números"; "Incapacidade para estabelecer uma correspondência recíproca"; "Escassa habilidade para contar compreensivamente"; "Dificuldade na compreensão dos conjuntos"; "Dificuldade na conservação"; "Dificuldade no cálculo"; "Dificuldade na compreensão do conceito de medida"; "Dificuldade para aprender a dizer as horas"; "Dificuldade na compreensão do valor das moedas"; "Dificuldade na compreensão da linguagem matemática e dos símbolos"; "Dificuldade em resolver problemas orais." (VIEIRA, 2004, p. 116).
}

Para Lara (2004), um estudante, mesmo discalcúlico, pode apresentar um bom desempenho em outras áreas do conhecimento e, até mesmo ao término do ano letivo, atingir a aprovação. Isso se deve ao fato de que apenas algumas áreas do cérebro são afetadas, em particular aquelas responsáveis pelas habilidades matemáticas, estando preservadas outras partes do cérebro encarregadas pelas demais habilidades cognitivas.

Nesse sentido, Lara (2004) ressalta a necessidade do professor identificar as diferenças entre as DAM e a Discalculia, ou, ao menos, suspeitar de estudantes que podem apresentar indícios desse transtorno e informar aos setores pedagógicos da escola, de modo que possam fazer os encaminhamentos necessários para a avaliação psicológica, psicopedagógica e neurológica. Desse modo, terão subsídios para oportunizar a esses estudantes as intervenções necessárias, a fim de resgatar as habilidades prejudicadas e minimizar os problemas vivenciados pelo estudante.

\subsection{Procedimentos de avaliação}

Na concepção de Bossa (2005), o diagnóstico psicopedagógico torna-se fundamental, pois propicia um olhar cuidadoso acerca da problemática da aprendizagem, objeto de estudo da psicopedagogia, no intento de compreender "por que e como" uma criança não aprende.

Diante disso, Weiss (2005) aponta que:

Somente uma boa avaliação psicopedagógica [...] de uma criança pode discernir e ponderar devidamente "o que" e "o quantum" é da criança, da escola, da família e da interação constante dos três vetores na construção das dificuldades de aprendizagem apontadas pela escola (WEISS, 2005, p. 180).

Entre os critérios para o diagnóstico de TAM, García (1998) destaca que o rendimento é abaixo do esperado para a escolarização e para a capacidade intelectual do sujeito, em testes 
padronizados. As dificuldades apresentadas interferem, significativamente, nas aprendizagens escolares, refletindo em atividades cotidianas.

A esse respeito, Haase et al. (2011) ressaltam que:

Para o diagnóstico de transtorno específico de aprendizagem (TEA), é necessário que as dificuldades sejam crônicas, persistindo de uma série para a outra, de uma avaliação para a próxima [...] é necessário excluir deficiências intelectuais inespecíficas, dificuldades emocionais ou falta de experiência cultural e/ou de aprendizagem adequada. O critério comportamental se baseia no desempenho de algum teste específico e padronizado de rendimento escolar, como, por exemplo, o Teste de desempenho escolar - TDE [...] (HAASE et al., 2011).

Haase et al. (2011) destacam a necessidade de pesquisadores se interessarem em elaborar instrumentos diagnósticos, com a intenção de diagnosticarem com eficácia o TAM, da mesma maneira que buscou-se compreender a Dislexia, elaborando instrumentos e intervenções capazes de atender as defasagens relacionadas ao transtorno. Corroborando, Bastos (2006) menciona que se faz necessário que os profissionais da Educação compreendam que as crianças e adolescentes com DAM deixem de ser considerados como desinteressados em se tratando da disciplina de Matemática. E sim, faz-se necessário proporcionar uma atenção diferenciada aos estudantes com dificuldades.

Segundo WEISS (2004), o período do diagnóstico pode ser denominado como uma investigação da não aprendizagem do sujeito ou da DA, a qual proporcionará ao terapeuta levantar hipóteses provisórias que poderão, ou não, ser confirmadas no decorrer do processo de intervenção.

\section{MAPA DAS PESQUISAS ACADÊMICAS}

Segundo Biembengut (2008), elaborar um mapa teórico é realizar uma revisão da literatura disponível sobre determinado tema a ser investigado e, também, das pesquisas acadêmicas, as quais estão sendo estudadas nos últimos anos, propiciando vastos conhecimentos referentes à área pesquisada.

\subsection{Identificação}

No intuito de realizar o mapeamento para o presente artigo, utilizou-se os dados disponibilizados nos sites CAPES e SCIELO, nos quais foi possível verificar os artigos de periódicos brasileiros publicados nos últimos anos, utilizando-se as palavras-chave: Discalculia; transtornos de aprendizagem em Matemática; dificuldades de aprendizagem em Matemática.

Primeiramente, foi digitada a palavra Discalculia, obtendo-se 7 artigos de periódicos no site da SCIELO e 9 artigos de periódicos no site da CAPES. Posteriormente, buscou-se pelas palavras transtornos de aprendizagem em Matemática, obtendo-se 2 artigos de periódicos 
na SCIELO e 35 na CAPES. A busca foi finalizada, utilizando-se as palavras dificuldades de aprendizagem em Matemática, resultando em 21 artigos de periódicos na SCIELO e 218 na CAPES.

Para explicitar os resultados encontrados nessa primeira busca, elaborou-se o gráfico apresentado na Figura 1.

\section{Figura 1 - Relação dos artigos publicados em periódicos brasileiros sobre Discalculia - DC, DAM e TAM}

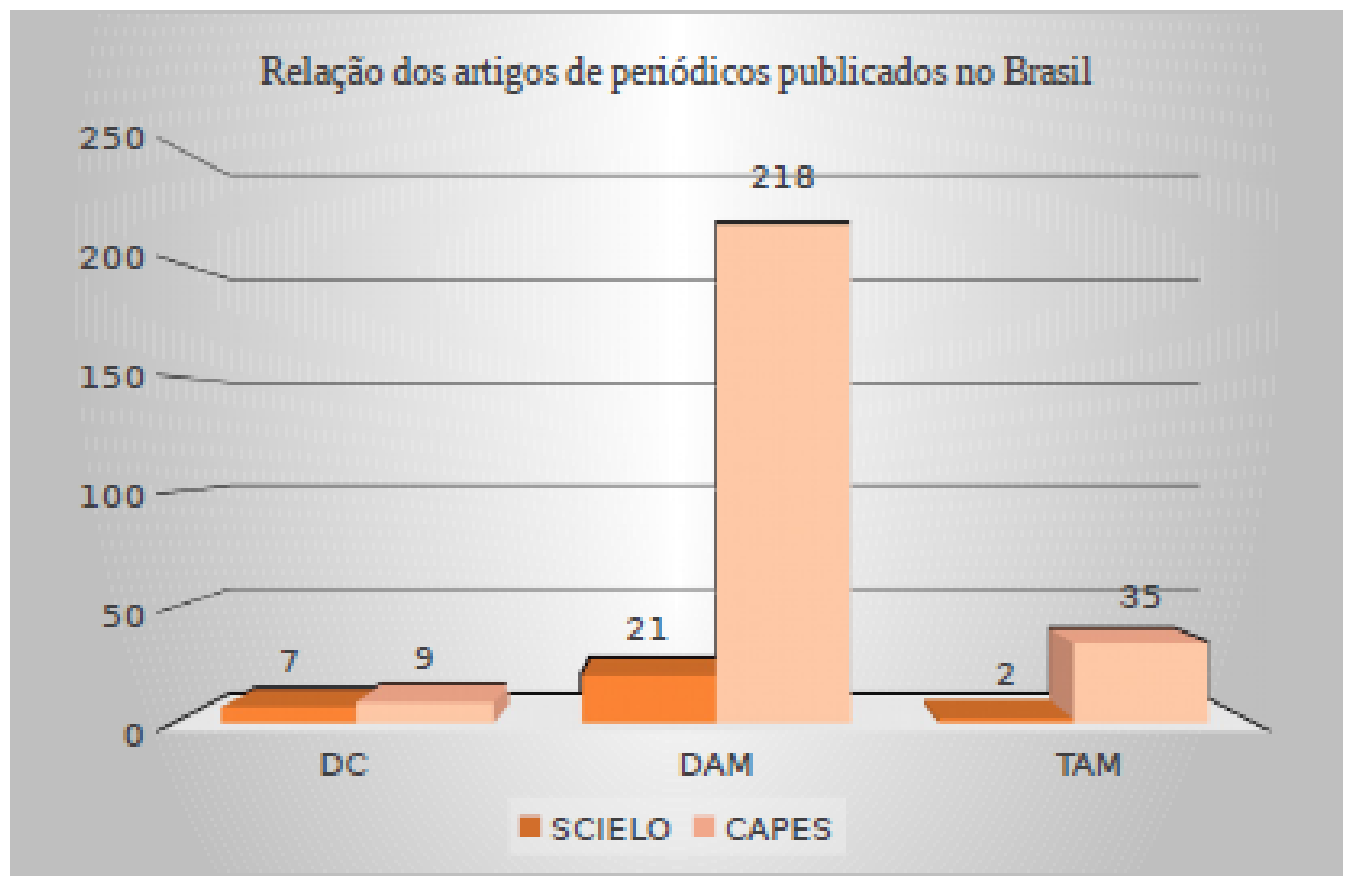

Fonte: Elaborada pelas autoras, a partir dos dados fornecidos pelos sites SCIELO e CAPES.

O gráfico explicita que existe um número maior de produções relativas às DAM e aos TAM em relação à Discalculia. Com base na leitura dos resumos dos artigos, percebeu-se que, especificamente sobre Discalculia, o número de artigos em periódicos brasileiros é extremamente restrito e relacionado ao transtorno de déficit de atenção e hiperatividade (TDAH).

Em se tratando da busca realizada, não foi delimitado um período, optou-se por levar em conta as três palavras-chave. Após a leitura dos resumos dos artigos de periódicos, disponíveis nos sites da CAPES e da SCIELO, foram selecionados 10 artigos para serem lidos na íntegra, preocupando-se em escolher os relacionados ao tema de pesquisa, Discalculia.

\subsection{Classificação e organização}

Para apresentar as produções selecionadas, elaborou-se o Quadro 1, constando o ano, os autores e o título de cada produção. 
Quadro 1 - Dados sobre os 10 artigos de periódicos selecionados

\begin{tabular}{|c|c|c|}
\hline Ano & Autor(es) & Título \\
\hline 2011 & $\begin{array}{l}\text { Jussara Bernardi; Claus } \\
\text { Dieter Stobaus }\end{array}$ & Discalculia: conhecer para incluir \\
\hline 2011 & $\begin{array}{l}\text { Paulo Adilson Silva; } \\
\text { Flávia Heloísa Santos }\end{array}$ & $\begin{array}{l}\text { Discalculia do desenvolvimento: avaliação } \\
\text { da representação umérica pela ZAREKI-R }\end{array}$ \\
\hline 2010 & $\begin{array}{l}\text { Paulo Adilson Silva; } \\
\text { Flávia Heloísa Santos }\end{array}$ & Mau desempenho escolar: uma visão atual \\
\hline 2008 & $\begin{array}{l}\text { Dalva Alice } \text { Rocha } \\
\text { Mol; Solange } \text { Muglia } \\
\text { Wechsler }\end{array}$ & $\begin{array}{l}\text { Avaliação de crianças com indicação de } \\
\text { dificuldades de aprendizagem pela bateria } \\
\text { Woodcock-Johnson III }\end{array}$ \\
\hline 2009 & $\begin{array}{l}\text { Maria de Nazaret Trin- } \\
\text { dade }\end{array}$ & $\begin{array}{l}\text { As Dificuldades de Aprendizagem em Lei- } \\
\text { tura e Aritmética: indicações de um estudo } \\
\text { piloto }\end{array}$ \\
\hline 2012 & $\begin{array}{l}\text { Luciana Vellinho } \\
\text { Corso; Beatriz Vargas } \\
\text { Dorneles }\end{array}$ & $\begin{array}{l}\text { Qual o papel que a memória de trabalho } \\
\text { exerce na aprendizagem da matemática? }\end{array}$ \\
\hline 2012 & $\begin{array}{l}\text { Vitor Geraldi Haase; } \\
\text { Annelise Júlio Costa; } \\
\text { Andressa Moreira An- } \\
\text { tunes; Isabella Starling } \\
\text { Alves }\end{array}$ & $\begin{array}{l}\text { Heterogeneidade Cognitiva nas Dificuldades } \\
\text { de Aprendizagem da Matemática: Uma Re- } \\
\text { visão Bibliográfica }\end{array}$ \\
\hline 2002 & $\begin{array}{lr}\text { Alexandra } & \text { Prufer } \\
\text { de } \quad \text { Queiroz } & \text { Campos } \\
\text { Araújo } & \end{array}$ & $\begin{array}{l}\text { Avaliação e manejo da criança com dificul- } \\
\text { dade escolar e distúrbio de atenção. }\end{array}$ \\
\hline 2012 & $\begin{array}{l}\text { Adriana Corrêa Costa; } \\
\text { Beatriz Vargas Dorne- } \\
\text { les; Luiz Augusto Paim } \\
\text { Rohde }\end{array}$ & $\begin{array}{l}\text { Identificação dos procedimentos de conta- } \\
\text { gem e dos processos de memória em crian- } \\
\text { ças com TDAH }\end{array}$ \\
\hline 2012 & $\begin{array}{l}\text { Adriana Corrêa Costa; } \\
\text { Luis Augusto Rohde; } \\
\text { Beatriz Vargas Dornele }\end{array}$ & $\begin{array}{l}\text { Desenvolvimento de fatos numéricos em es- } \\
\text { tudantes com transtornos de aprendizagem }\end{array}$ \\
\hline
\end{tabular}

Fonte: Elaborado pelas autoras, com base nos dados fornecidos pelos sites SCIELO e CAPES.

Ao realizar a leitura dos respectivos resumos dos artigos escolhidos, observou-se que muitos não tratavam especificamente da Discalculia, mesmo a busca tendo partido de um assunto específico e sido utilizadas as palavras-chave destacadas anteriormente. As pesquisas em um mesmo artigo tratavam não somente da Discalculia, como das DAM e dos distúrbios de aprendizagem.

Na intenção de organizar esses assuntos, apresenta-se na Figura 2 os termos mencionados nos resumos. 


\section{Figura 2 - Termos mencionados nos resumos}

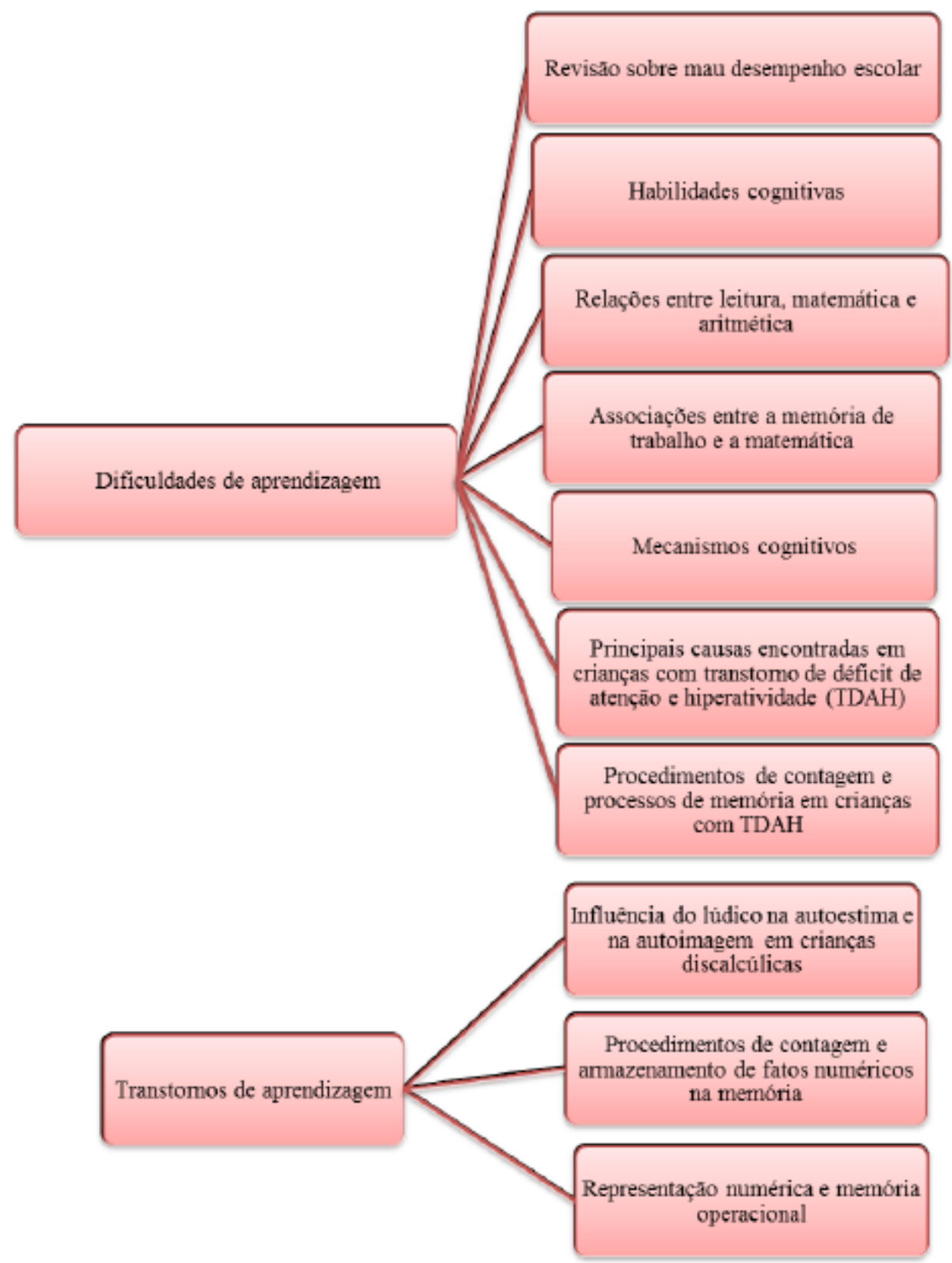

Fonte: Elaborada pelas autoras, com base nos dados fornecidos pelos sites SCIELO e CAPES.

Para realizar o reconhecimento e a análise dos artigos selecionados, elaborou-se uma síntese de cada artigo, detalhados, a seguir, a partir dos aspectos analisados mencionados anteriormente.

Em relação ao artigo, "Discalculia conhecer para incluir", Bernardi e Stobäus (2011) apontam como objetivo verificar a influência do lúdico na autoestima e autoimagem de participantes discalcúlicos. Referente aos procedimentos metodológicos, a pesquisa foi realizada com uma abordagem qualitativo-quantitativa com cinco participantes que foram encaminhados ao laboratório de aprendizagem por apresentarem dificuldades relacionadas ao conhecimento matemático. Em se tratando das contribuições, destaca-se que a utilização de constantes intervenções pedagógicas, aos alunos com Discalculia, pode auxiliar em suas dificuldades, melhorar a autoestima, a autoimagem e a motivação para aprender.

No artigo "Discalculia do Desenvolvimento: avaliação da representação numérica pela 
ZAREKI-R”, Silva e Santos (2011) estudaram os aspectos da representação numérica e da memória operacional de crianças com TA. Metodologicamente, foi realizada a pesquisa de abordagem qualitativo-quantitativa, utilizando a ZAREKI-R como instrumento para a avaliação da Discalculia do Desenvolvimento (DD). Como contribuições, salienta-se que os sujeitos com déficits específicos em memória operacional visuoespacial e comprometimento em processamento numérico e cálculo são compatível com DD.

Em se tratando do artigo "Mau desempenho escolar: uma visão atual", Siqueira e Giannetti (2011) tiveram como objetivo realizar uma revisão atualizada sobre o tema mau desempenho escolar. Como procedimentos metodológicos, foi realizada a pesquisa de abordagem qualitativa com ênfase na análise do conteúdo, por meio de revisão da literatura sobre aprendizagem, desempenho escolar, TA, TDAH e transtorno de desenvolvimento de coordenação (TDC). Em relação às contribuições, menciona-se o fator mau desempenho escolar como um sintoma frequentemente nas crianças, o qual tende a afetar gravemente as questões emocionais, sociais e econômicas.

No artigo "Avaliação de crianças com indicação de dificuldades de aprendizagem pela bateria Woodcock-Johnson III", Mól e Wechsler (2008) investigaram as habilidades cognitivas de crianças com indicação de DA, encaminhadas por seus professores. Trata-se de uma pesquisa de abordagem qualitativo-quantitativa, utilizando-se da Bateria de Habilidades Cognitivas Woodcock-Johnson III - WJ III, composta por 10 testes e as notas escolares em Português, Matemática e Ciências. Como contribuições dessa pesquisa, a mesma estende-se aos psicólogos escolares brasileiros, os quais necessitam orientar os professores quanto à identificação e à intervenção adequada junto aos alunos que estejam apresentando DA.

Trindade (2009), em seu artigo "As Dificuldades de Aprendizagem em Leitura e Aritmética: indicações de um Estudo Piloto", teve como objetivo investigar a existência de possíveis relações entre as dificuldades em leitura e Matemática, realizando uma pesquisa de abordagem qualitativo-quantitativa, usando-se um dispositivo experimental de investigação elaborado pela autora. Quanto às contribuições, vale mencionar que a identificação de semelhanças em nível da investigação e das teorias subjacentes e consequente aplicação podem fornecer ligações que conduzam ao aproveitamento dos avanços teóricos conseguidos no primeiro campo e à identificação de linhas convergentes entre os domínios da leitura e da aritmética, que possam levar a uma maior coerência teórica ao nível das DA.

No artigo "Qual o papel que a memória de trabalho exerce na aprendizagem da Matemática?", Corso e Dorneles (2012) apontaram como objetivo verificar se as DAM estão associadas a defasagens em componentes específicos da memória de trabalho, ou estão relacionadas ao déficit geral desse sistema, considerando o modelo de Baddeley e Hicht. Quanto aos procedimentos metodológicos, foi realizada a pesquisa de abordagem qualitativa com ênfase na análise de conteúdo, apresentando o modelo de memória de trabalho proposto por Baddeley e Hitch (1974), caracterizando os três componentes que formam a memória de trabalho. Posteriormente, realizaram uma revisão da literatura, discutindo se as DAM estão associadas a defasagens em componentes específicos da memória de trabalho, ou estão relacionadas ao défi- 
cit geral desse sistema. Após essa etapa, foi analisada a diversidade encontrada nos resultados das pesquisas acerca da memória de trabalho e as DAM, apontando possíveis justificativas para tal situação. Feito isso, apresentou-se diferentes dados de pesquisa analisando se os prejuízos na memória de trabalho são causa ou consequência das DAM. Convém destacar que as contribuições do respectivo artigo referem-se à possibilidade que o suporte à memória de trabalho, por meio de programas de intervenção, podem proporcionar melhores resultados acadêmicos não só na Matemática, como nas mais diversas áreas do currículo.

Quanto ao artigo "Heterogeneidade Cognitiva nas Dificuldades de Aprendizagem da Matemática: Uma Revisão Bibliográfica”, Haase et al. (2012) analisaram, por meio de uma revisão de literatura, os possíveis mecanismos cognitivos envolvidos nas DAM e em outros distúrbios relacionados. Trata-se de uma pesquisa de abordagem qualitativa com ênfase na análise de conteúdo dos possíveis mecanismos cognitivos envolvidos nas DAM e em outros distúrbios relacionados. Pode-se destacar como contribuições que os diagnósticos e intervenções neuropsicológicos melhoram o funcionamento cognitivo dos indivíduos. Conclui-se que o processamento fonológico, a memória de trabalho, o processamento visuoespacial e o senso numérico são domínios potencialmente subjacentes às DAM.

Araújo (2002), no artigo "Avaliação e manejo da criança com dificuldade escolar e distúrbio de atenção", teve como objetivo oferecer aos pediatras as principais causas encontradas em crianças com dificuldade escolar, com enfoque na avaliação e na conduta dos casos associados ao TDAH. Foi realizada a pesquisa de abordagem qualitativa com ênfase na análise do conteúdo, baseando-se, em uma revisão da literatura médica, a partir de levantamento de artigos no Medline, a partir da busca pelo PubMed, nos últimos 5 anos, com as palavras chave de TA, TDAH, Dislexia e Discalculia. Em se tratando das contribuições, é válido salientar que o tratamento medicamentoso com estimulantes, antidepressivos ou clonidina, associado ao acompanhamento multidisciplinar, proporciona o melhor desempenho das crianças com TDAH.

Em relação ao artigo "Identificação dos procedimentos de contagem e dos processos de memória em crianças com TDAH”, Costa et al. (2012b) tiveram como objetivo principal identificar os procedimentos de contagem e os processos de memória utilizados por um grupo de estudantes com diagnóstico de TDAH. Como procedimentos metodológicos, foi realizada uma pesquisa de abordagem qualitativo-quantitativa com 28 estudantes de escolas públicas da cidade de Porto Alegre, de ambos os sexos, com idades entre 8 e 14 anos. A composição da amostra teve três fases: fase 1 - Triagem no banco de dados; fase 2 - Convite por meio de contato telefônico e telegrama; fase 3 - Avaliação dos procedimentos de contagem. As contribuições observadas estão associadas ao armazenamento e ao acesso automático dos fatos aritméticos da memória de longo prazo, os quais têm sido apontados como habilidades prejudicadas em crianças com TDAH. Estudantes com TDAH utilizam procedimentos de contagem mais imaturos do que seus pares sem dificuldades.

No artigo "Desenvolvimento de fatos numéricos em estudantes com transtornos de aprendizagem”, Costa et al. (2012b) apresentaram uma revisão do tema sobre a representação dos fatos numéricos aditivos na memória de longo prazo em estudantes com TAM. Como procedi- 
mentos metodológicos, foi realizada a pesquisa de abordagem qualitativa com ênfase na análise do conteúdo, um exame abrangente da literatura indicando que as crianças com TAM utilizam procedimentos de contagem já abandonados por seus iguais da mesma idade, e apresentam desenvolvimento tardio ou atípico na recuperação e armazenamento dos fatos numéricos na memória. Como contribuições, enfatizam que o ensino de fatos básicos aditivos, não tem recebido a devida importância e que o ensino continua a enfatizar a memorização, ao invés da compreensão Matemática, o que pode acabar dificultando essa automatização, pelo menos para estudantes com DAM.

\subsection{Reconhecimento e análise}

Conforme Biembengut (2008, p. 95), "Reconhecer significa identificar e assinalar concepções teóricas e principais resultados. Analisar implica combinar vários dados ou resultados específicos em um mais geral, realizando combinações por meio de associações em função de similares [...]." Assim, após realizar a síntese de cada artigo, busca-se, nessa seção, analisar convergências e divergências entre os mesmos, classificando-os e agrupando-os.

Com base na leitura integral de cada um dos artigos, foi possível identificar a utilização de distintos termos para referir-se às DA e aos TA, sejam eles: Discalculia (3) ${ }^{8}$; DAM (4); TAM (1); e distúrbios de aprendizagem (2). Evidencia-se uma diversidade de termos, o que acaba por dificultar a comparação entre os dados pesquisados, pela falta de padronização das denominações utilizadas. Vale sublinhar que tais termos possuem sentidos diferentes, como descrito anteriormente.

Diante disso, no caso dos artigos selecionados para este mapeamento, verifica-se que dedicam-se a investigações distintas, o que contribui para que existam diferentes abordagens e informações acerca desse transtorno.

Em se tratando dos objetivos, observou-se que cada uma das produções investigam ou dedicam-se a propósitos diferentes, com exceção do objetivo de apresentar uma revisão da literatura acerca das DA e dos TA encontrado em 2 artigos. Fica visível que para definir Discalculia os pesquisadores escolheram autores que vão ao encontro do objetivo de seu estudo.

Costa et al. (2012a) referem-se a Mazzocco (2007) e Murphy et al. (2007), os quais salientam que o termo transtorno (disability) remete-se a uma condição determinada biologicamente, estando o aluno abaixo do desempenho em Matemática, se comparado por colegas da mesma faixa etária e escolaridade.

Araújo (2002) define Discalculia, a partir do DSM-IV, o qual refere-se ao transtorno como havendo dificuldade em operar os conceitos matemáticos.

Bernardi e Stobäus (2011) explicam o conceito de Discalculia fazendo referência a Kosc (1974), o qual explica o termo como sendo uma desordem estrutural nas habilidades matemáticas, com origem em questões genéticas ou congênitas em determinadas partes do cérebro.

\footnotetext{
${ }^{8} \mathrm{O}$ numeral apresentado entre parênteses representa o número de artigos que foram identificados no respectivo item.
} 
Silva e Santos (2011) conceituam o TAM, apontando os dados do DSM-IV-TR e do CID10, em que a Discalculia do Desenvolvimento é denominada como um transtorno específico, o qual afeta as habilidades cognitivas da Matemática em sujeitos com inteligência normal.

Haase et al. (2012) mencionam as contribuições de Mazzocco (2007) ao salientar, em sua pesquisa, que os sujeitos com Discalculia tendem a apresentar dificuldades mais graves, as quais são persistentes ao longo da vida.

Em relação aos procedimentos metodológicos adotados nessas produções, percebeu-se a prevalência da abordagem qualitativa com ênfase na análise de conteúdo (5) e da abordagem qualitativo-quantitativa (5).

Foi possível verificar algumas considerações relevantes salientadas nas produções selecionadas, entre elas: crianças com TA apresentam prejuízos significativos quanto a memória operacional (3), crianças com distúrbios de aprendizagem não conseguem aprender de forma global ou específica, devido às disfunções no SNC (2); entre os diversos processos cognitivos que podem justificar as DAM, há um conjunto de evidências empíricas que apontam a memória de trabalho como um fator importante (1); no TAM, existe dificuldade em operar conceitos matemáticos (1); as DAM consistem em uma condição que altera a aquisição das habilidades aritméticas (1); o aprendiz em aritmética se apoia, inicialmente, em estratégias de processamento para resolver problemas, mas com a idade e o aumento da competência, passa a usar a retenção direta de fatos aritméticos (1); o mau desempenho escolar é um sintoma em nossas escolas com graves repercussões emocionais, sociais e econômicas (1).

A partir disso, destacam-se como principais contribuições: problemas na memória de trabalho repercutem em situações cotidianas, as quais estão envolvidas tarefas matemáticas, dificultando a aprendizagem dos alunos (2); a necessidade dos psicólogos orientarem os professores na identificação e na intervenção de alunos com DA (1); estudantes com TDAH apresentam atraso no desenvolvimento dos procedimentos de contagem, e não um desvio, quando comparados a sujeitos com desenvolvimento típico (1); noções atualizadas das principais causas das dificuldades escolares, com enfoque mais detalhado na avaliação e na conduta dos casos associados ao TDAH (1); a neuropsicologia pode colaborar no desenvolvimento de instrumentos diagnósticos e auxiliar a planejar estratégias eficientes para a intervenção (1); intervenções pedagógicas adequadas pelos professores podem propiciar aos alunos com Discalculia superarem suas dificuldades, melhorando a autoestima, a autoimagem e a motivação para aprender (2); a necessidade dos profissionais de Educação e Saúde estarem habilitados a identificar as crianças em risco de dificuldades em aprender, orientar os familiares e se necessário encaminhar para a reabilitação multidisciplinar, na busca de um diagnóstico condizente, possibilitando a aprendizagem de estratégias oportunas e adequadas precocemente (2).

Contudo, de acordo com a DA ou o TA, nem sempre o trabalho do professor consegue dar conta das dificuldades, às vezes é preciso o encaminhamento, junto ao setor pedagógico, aos profissionais especializados, bem como: psicopedagogo; psicólogo; psiquiatra; neurologista; fonoaudiólogo, para realização de um trabalho multidisciplinar com a escola, a família e o estudante (ROTTA, 2006). 


\section{CONSIDERAÇÕES FINAIS}

Este estudo teve como objetivo realizar um mapeamento dos artigos publicados em periódicos, buscando relações entre o modo como são conceituados os termos Discalculia, TAM e DAM e as contribuições aos profissionais da área da Educação e da Saúde, sem período de tempo estipulado, a partir da consulta ao banco de periódicos disponibilizados pela CAPES e pela SCIELO.

A partir da leitura integral dos artigos selecionados nesse mapeamento, foi possível classificar e agrupar as produções por meio da síntese das ideias fundamentais de cada uma delas, trazendo à tona alguns dos principais aspectos do estudo em questão.

Verifica-se, assim como Pimentel e Lara (2013), que alguns artigos utilizam indistintamente os termos Discalculia, DAM, TAM e distúrbios de aprendizagem para referir-se às DA e aos TA, o que ocasiona uma dificuldade na comparação entre os dados pesquisados, pela falta de padronização e, até mesmo, coerência quanto às denominações utilizadas.

Em relação à fundamentação teórica nos artigos, poucos artigos citaram os mesmos autores, como Van de Walle, Costa, Dorneles, Baddeley e Hitch e Geary. Sugere-se que isso se deva ao fato de adotarem diferentes terminologias da literatura brasileira para designar DA e TA.

Verificou-se, por meio da análise das pesquisas, que metade dos artigos utilizou a abordagem qualitativa com ênfase na análise de conteúdo e, o restante, a abordagem qualitativoquantitativa. Embora com diferentes instrumentos de coleta de dados, percebe que os procedimentos metodológicos foram os mais adequados a cada tipo de investigação.

Na busca pela compreensão do termo Discalculia, foi possível constatar que a maioria dos artigos aponta que se trata de um transtorno de aprendizagem que afeta as habilidades matemáticas específicas, sob as quais necessita-se de um olhar minucioso do psicopedagogo. Contudo, a falta de instrumentos de avaliação capazes de proporcionarem com precisão que tipo de habilidade está em defasagem e a escassez de literatura sobre esse transtorno, dificulta a atividade desse profissional, impedindo-o, muitas vezes, de realizar intervenções que de fato reabilitem as habilidades debilitadas.

Vale ressaltar que se os artigos selecionados fossem outros, outras considerações certamente seriam tecidas. No entanto, as conclusões parciais que aqui se evidenciam chamam a atenção para a necessidade de estudos voltados à questão da avaliação e de intervenções psicopedagógicas que possam ser desenvolvidas com crianças e adolescentes com indícios de Discalculia. Essas preocupações já vêm sendo abordadas pelo GEPED/PUCRS, em particular nos estudos de Pimentel (2015) e Avila (2017). 


\section{REFERÊNCIAS}

ARAÚJO, A. P. Q. C. Avaliação e manejo da criança com dificuldade escolar e distúrbio de atenção. Jornal de Pediatria, Scielo, v. 78, p. 104 - 110, jul. 2002.

AVILA, L. A. B. Avaliação e intervenções psicopedagógicas em crianças com indícios de discalculia. 2017. 281 f. Dissertação (Mestrado) — Pontifícia Universidade Católica do Rio Grande do Sul, Rio Grande do Sul, 2017.

BASTOS, J. A. Discalculia: transtorno específico da habilidade em matemática. In: ROTTA, N.;OHLWEILER, L.; RIESCO, R. (ORGS). Transtornos da aprendizagem: Abordagem neurobiológica e multidisciplinar. São Paulo: Artes Médicas, 2006. p. 195-206.

BERNARDI, J.; STOBÄUS, C. D. Discalculia: conhecer para incluir. Revista Educação Especial, v. 24, n. 39, p. 47-60, jan/abril 2011.

BIEMBENGUT, M. S. Mapeamento na pesquisa educacional. Rio de Janeiro: Ciencia Moderna, 2008.

BOSSA, N. A. Introdução: Avaliação psicopedagógica da criança de 7 a 11 anos. In: BOSSA, N. A.; OLIVEIRA, V. B. (ORGS). Avaliação psicopedagógica da criança de sete a onze anos. Petrópolis: Vozes, 2005. p. 07-14.

BUCHWEITZ, A. ACERTA - Avaliação de Crianças em Risco de Transtorno de Aprendizagem. 2015. <http://www3.pucrs.br/portal/page/portal/inscer/Capa/ACERTA > . Acesso em: 18 de maio 2015.

BUTTERWORTH, B. Developmental dyscalculia. In: CAMPBELL, J. I. D (ED.). Handbook of Mathematical. New York: Psychology, 2005. p. 455-467.

CORSO, L. V.; DORNELES, B. V. Qual o papel que a memória de trabalho exerce na aprendizagem da matemática? Boletim: Boletim de Educação Matemática, v. 26, n. 42, p. 627-648, abr. 2012.

COSTA, A. C.; DORNELES, B. V.; ROHDE, L. A. Desenvolvimento de fatos numéricos em estudantes com transtornos de aprendizagem. Bolema: Boletim de Educação Matemática, v. 26, n. 44, p. 1151-1170, dez. 2012.

COSTA, A. C.; DORNELES, B. V.; ROHDE, L. A. Identificação dos procedimentos de contagem e dos processos de memória em crianças com TDAH. Psicologia e Reflexão Crítica, v. 25, n. 4, p. 791-801, 2012.

DSM-5. Manual diagnóstico e estatístico de transtornos mentais. 5. ed. Porto Alegre: Artes Médicas, 2014.

FERNÁNDEZ, A. O saber em jogo. Porto Alegre: Artes Médicas, 2001.

FONSECA, V. Abordagem psicopedagógica das dificuldades de aprendizagem. 2. ed. Lisboa: Âncora, 2000.

GARCÍA, J. N. Manual de dificuldades de aprendizagem: linguagem, leitura, escrita e matemática. Porto Alegre: Artes Médicas, 1998.

HAASE, V. G. et al. Heterogeneidade cognitiva nas dificuldades de aprendizagem da matemática: uma revisão bibliográfica. Psicologia em pesquisa, v. 6, n. 2, p. 139-150, dez. 2012. 
HAASE, V. G. et al. O estatuto nosológico da discalculia do desenvolvimento. In: CAPOVILLA, F. C. (ORG). Transtornos de aprendizagem 2: Da análise laboratorial e da reabilitação clínica para as políticas públicas de prevenção pela via da educação. São Paulo: Memnon Edições Científicas, 2011. p. 139-144.

ISLEY, P. Organização Mundial de Saúde - Classificação Estatística Internacional de Doenças e Problemas Relacionados à Saúde. 2015. Disponível em: <http://www.datasus.gov.br/ cid10/V2008/cid10.htm>. Acesso em 19 de maio de 2015.

KOSC, L. Developmental dyscalculia. Journal of learning disabilities, v. 7, n. 3, p. 163-177, mar. 1974.

LARA, I. C. M. Ensino inadequado de matemática. Revista Ciências e Letras, n. 35, p. 137 152, mar./jul. 2004.

MACHADO, N. J. Matemática e Realidade. 2. ed. São Paulo: Cortez, 1997.

MÓL, D. A. R.; WECHSLER, S. M. Avaliação de criançaas com indicação de dificuldades de aprendizagem pela bateria Woodcock-Johnson III. Revista Semestral da Associação Brasileira de Psicologia Escolar e Educacional (ABRAPEE), v. 12, n. 2, p. 391-399, dez. 2008.

MYKLEBUST, H. R.; JOHNSON, D. J. Dyslexia in children. Exceptional children, p. 14-25, 1962.

OHLWEILER, L. Introdução. In: ROTTA, N. T.;OHLWEILER, L.; RIESCO, R. S. (ORGS). Transtornos da aprendizagem: Abordagem neurobiológica e multidisciplinar. São Paulo: Artes Médicas, 2006. p. 127-130.

PIMENTEL, L. S. Discalculia e Provinha Brasil: uma análise do desempenho de estudantes do primeiro ano. 2015. 162f. Dissertação (Mestrado) - Pontifícia Universidade Católica do Rio Grande do Sul, Rio Grande do Sul, 2015.

PIMENTEL, L. S.; LARA, I. C. M. Discalculia: mapeamento das produções brasileiras. In: VI CONGRESSO INTERNACIONAL DE ENSINO DE MATEMÁTICA. Acta Latinoamericana de Matemática Educativa. Canoas: ULBRA, 2013.

RELVAS, M. P. Neurociências e transtornos de aprendizagem: as múltiplas eficiências para uma educação inclusiva. Rio de Janeiro: Wak ED, 2011.

ROTTA, N. T. Dificuldades para a aprendizagem. In: ROTTA, N.;OHLWEILER, L.; RIESCO, R. (ORGS). Transtornos da aprendizagem: Abordagem neurobiológica e multidisciplinar. São Paulo: Artes Médicas, 2006. p. 113-123.

SILVA, P. A.; SANTOS, F. H. Discalculia do desenvolvimento: avaliação da representação numérica pela ZAREKI-R. Psicologia: Teoria e Pesquisa, v. 27, n. 2, p. 169-177, abr./jun. 2011.

SIQUEIRA, C. M.; GIANNETTI, J. G. Mau desempenho escolar: uma visão atual. revista da associação médica brasileira. Revista da Associação Médica Brasileira, n. 1, p. 78-87, out. 2011.

TRINDADE, M. N. As dificuldades de aprendizagem em leitura e aritmética: indicações de um estudo piloto. Bolema: Boletim de Educação Matemática, n. 32, p. 61-81, mai. 2009.

VIEIRA, E. Transtornos na aprendizagem da matemática: Número e discalculia. Revista Ciências e Letras, n. 35, p. 109-120, mar./jul. 2004. 
WEISS, M. L. L. Psicopedagogia Clínica: uma visão diagnóstica dos problemas de aprendizagem escolar. 10. ed. Rio de Janeiro: DP\&A, 2004.

WEISS, M. L. L. A avaliação e a instituição escolar. In: BOSSA, N. A.; OLIVEIRA, V. B. (ORGS). Avaliação psicopedagógica da criança de sete a onze anos. Petrópolis: Vozes, 2005. p. $163-182$. 